\title{
Reliable Misrepresentation and Teleosemantics
}

\author{
Marc Artiga \\ Universitat de Girona - LOGOS \\ DOI: $10.2478 /$ disp-2013-0020 \\ BIBLID [0873-626X (2013) 37; pp. 265-281]
}

\begin{abstract}
Mendelovici (forthcoming) has recently argued that (1) tracking theories of mental representation (including teleosemantics) are incompatible with the possibility of reliable misrepresentation and that (2) this is an important difficulty for them. Furthermore, she argues that this problem commits teleosemantics to an unjustified a priori rejection of color eliminativism. In this paper I argue that (1) teleosemantics can accommodate most cases of reliable misrepresentation, (2) those cases the theory fails to account for are not objectionable and (3) teleosemantics is not committed to any problematic view on the color realism-antirealism debate.
\end{abstract}

\section{Keywords}

Teleosemantics, Misrepresentation, Mental Content, Color Realism, Mendelovici.

In a recent paper, Mendelovici (forthcoming) ${ }^{1}$ has argued that it is possible for representational systems to reliably misrepresent certain properties and that tracking theories such as teleosemantics are incompatible with this possibility. That incompatibility is supposed to highlight a significant drawback of tracking theories. In this paper I would like to argue that one of the most popular tracking theories, teleosemantics, is immune to these criticisms.

The paper is organized in the following way. In the first part, I outline teleosemantics and the problem of reliable misrepresentation. Then, I argue that teleosemantics is compatible with most cases of reliable misrepresentation and that those cases teleoseman-

\footnotetext{
${ }^{1}$ Since at the moment of writing these lines Mendelovici's paper is only available online, I refer to the pages of the electronic version.

Disputatio, Vol. V, No. 37, November 2013

Received: 16/01/2013 Accepted: 04/04/2013
} 
tics rules out are not objectionable. In the final section I address the particular example of reliable misrepresentation Mendelovici has in mind, color anti-realism, and discuss whether teleosemantics is indeed committed to color realism, as she suggests.

\section{Teleosemantics and reliable misrepresentation}

Teleosemantics is a naturalistic theory of content. ${ }^{2}$ It is based on two key notions: function and sender-receiver structure.

First of all, the concept of function employed is the so called etiological notion, according to which functions are selected effects (Neander 1995). According to this approach, functions are effects of traits that played an important causal role in the process of selection of that trait. For instance, the function of kidneys is to filter wastes from blood, because kidneys were selected for filtering wastes. That was the effect that explains why organisms having kidneys were favored by natural selection.

Secondly, the sender-receiver structure is an abstract model employed in communication theory and signal detection theory. In short, a sender-receiver structure is composed of two systems, a sender (or 'producer'), which takes some input and produces a state, and a receiver (or 'consumer'), which takes this state as input and produces an effect.

Teleosemantics puts these two notions together in order to provide a naturalistic account of representation. In a nutshell, the idea is that representations are states that stand between two systems, a sender and a receiver, when they are endowed with certain etiological functions. The function of the sender is to produce a state $\mathrm{R}$ (the representation) when another state $\mathrm{P}$ obtains (the representatum). The function of the receiver is to produce an effect (e.g. a behavior) when state $\mathrm{R}$ is tokened.

Now, a key question in the debate on naturalistic theories is what determines the content of the representation. What determines the meaning of $\mathrm{R}$ ? The answer of mainstream teleosemantics is that $\mathrm{R}$

\footnotetext{
${ }^{2}$ In this paper I heavily rely on Millikan's version of teleosemantics, which is the most sophisticated and popular view. Nevertheless, most of the claims presented here could be easily accepted by many teleosemantic accounts.
} 
represents the state of affairs $\mathrm{P}$ that the consumer system has historically needed in order to perform its function successfully (Millikan 1993; Papineau 1993). In other words: if we look at the evolution of the sender-receiver mechanism, $\mathrm{P}$ is the feature that was required for the consumer to act in a fitness-enhancing way. At the very end, the presence of $\mathrm{P}$ is what explains that the whole representational system exists (Neander 2012).

Let me illustrate this theory with an example. Male chicken (Gallus Gallus domesticus) produce a characteristic call when they find food, which brings other chicken to that location (Evans and Evans 1999). According to teleosemantics, this call is a representation and means something like there is food around, since (1) it stands within a sender-receiver structure, composed by the male chicken (sender) and its fellows (receiver) and (2) the presence of food is what causally explains that the interpreting mechanism (the fellow chicks) performed their function successfully (moving to the location where the call is made and ingesting food). In other words: this representational mechanism was useful because signs correlated with the presence of food usually enough. So, the presence of food is what explains the selection of this sender-receiver structure, and hence it is the state of affairs represented by the call.

Now, Mendelovici's objection concerns what she calls 'reliable misrepresentation'. In contrast to standard criticisms of naturalistic theories of content such as Fodor's (1990) misrepresentation (or disjunction) problem, her argument grants that teleosemantics can account for occasional misrepresentation (Mendelovici: 7). The objection concerns systematic or reliable misrepresentation.

In a nutshell, the idea is that a state type $\mathrm{R}$ reliably misrepresents $P$ just in case tokens of $R$ represent instantiations of $P$, most of the time $\mathrm{P}$ is not instantiated and this representational relation is somehow robust (i.e. in similar circumstances $\mathrm{R}$ would still misrepresent instantiations of P). More precisely, Mendelovici (4) defines reliable misrepresentation as follows:

Reliable Misrepresentation

An organism's representation type R reliably misrepresents some property P iff: 
1. Some tokens of $\mathrm{R}$ are involved in attributive mental states that represent objects as having property $\mathrm{P}$,

2. Most or all of the relevant objects do not have $\mathrm{P}$,

3. Tokens of R do or would nonveridically represent objects as having $\mathrm{P}$ in the same types of circumstances on separate occasions.

Now, Mendelovici's argues that cases of reliable representation are possible but cannot be accommodated with teleosemantics. That is, she claims teleosemantics is incompatible with the possibility of cases of reliable misrepresentation. The reason is quite simple indeed: since teleosemantics claims that the representational content of $\mathrm{R}$ is determined by the state that existed in the past and had a causal influence in the selection of the representational system, it seems to be committed to the usual existence of the represented state. Only something that existed could have had this causal influence. But if the represented state has usually existed in the past, then the representation has been true most of the time. So there seems to be some tension between the teleosemantic view and the existence of systematic misrepresentation.

Mendelovici goes on to argue that this incompatibility with cases of reliable misrepresentation has important consequences for certain philosophical discussions, such as the color realism/anti-realism debate. If teleosemantics is incompatible with cases of reliable misrepresentation, then teleosemanticists cannot accept that our color representations are false most of the time. Consequently, Mendelovici concludes teleosemantics is committed to color realism. And that seems to be a problematic consequence because a general theory of representation such as teleosemantics should not be committed to any particular view on the realism/anti-realism debate. So the theory is in trouble.

I think this objection is worth exploring, not only because this reasoning is intuitively compelling, but also because naturalistic accounts have traditionally had a hard time accounting for the possibility of misrepresentation. In what follows, however, I would like to argue that teleosemantics is fully compatible with the possibility of 
most cases of reliable misrepresentation, and that those cases that cannot be accounted for are not problematic. In the final section, I will consider the relation between teleosemantics and the debate on color realism.

\section{Accounting for reliable misrepresentation}

The first part of Mendelovici's argument tries to settle that teleosemantics is incompatible with a set of possible cases that involve reliable misrepresentation. However, I think that, as stated, Reliable MisRepresentation clearly fails to pin down this set of situations. Indeed, some of the cases that abide by Reliable MisRepresentation are extremely common, and teleosemanticists usually recognize them (Millikan 1984, 1993; Neander 1995).

Cockroaches and crickets, for instance, possess a set of short appendages at the rear of their abdomen called 'cerci'. Cerci are slender filiform hairs sensitive to air movements. When air moves at a high speed and reaches a certain threshold, a set of neurons are automatically activated and cockroaches perform a range of evasive behaviors (Comer and Leung 2004). According to teleosemantics, this neuronal activation means something like there is a predator around, since it is the fact that in some cases a predator was around that explains that the utility of this evasive behavior, and hence accounts for the selection of the mechanism. When there was no predator, this behavior was just a waste of time and energy. But, crucially, notice that probably most of the time cerci produce a neuronal activation and hence an evasive behavior when there is no predator around. That means that the cockroach's neuronal states are probably false most of the time. That is not a problem for teleosemantics because it is still true that what explains the selection of the mechanism is the fact that there was a predator around usually enough. Natural selection only requires that in certain cases a trait provides a significant advantage; it does not require that this situation be the most common one.

This is not an isolated example. Whenever a false negative is much more significant than a false positive, signals will tend to be produced in many situations when in fact there is no threat (Godfrey-Smith 1996; Skyrms 2010). When a single false negative could be the last one, organisms tend to produce many false positives (Mil- 
likan 1993).

Crucially, notice that this representational system seems to fulfill all conditions set up in Reliable MisRepresentation. The representation $\mathrm{R}$ (in this case, certain neuronal activity) represents there being danger around (condition 1), in most cases there is no danger (condition 2) and in many similar circumstances R still misrepresents the presence of danger (condition 3). So cases that satisfy Reliable MisREPRESENTATION are perfectly compatible with teleosemantics.

In order for Mendelovici's argument to have some bite, she needs to exclude this sort of cases, which teleosemantics trivially accounts for. In particular, condition 2 should be restricted to representations of objects that never instantiate a certain property P. That is, clause 2 must be read as stating that 'all of the relevant objects do not have P'. Indeed, that version fits much better with the particular counterexamples Mendelovici brings forward in her paper. Let us call this modified definition 'STRONG Reliable MisRePRESENTATION'.

Is teleosemantics incompatible with cases that fulfil STRONG RELIable misRepresentation? Probably not. We can also describe some scenarios in which teleosemantics can satisfactorily accommodate STRONG RELIABLE MISREPRESENTATION.

Think first about organisms which are endowed with a mechanism for representing the size of objects. This mechanism will produce a different representation depending on the input it receives. Now, while it is extremely plausible that most of the time we represent the right size of objects, there is a vast literature in psychology describing cases in which this mechanism systematically yields inaccurate representations. For instance, in the best-known version of the Ebbinghaus illusion, two circles of identical size are placed near to each other and one is surrounded by large circles while the other is surrounded by small circles; the first central circle then appears smaller than the second central circle. ${ }^{3}$ In this case, the selection and existence of a mechanism producing representations of size is explained by the fact that most of the time it produces the right rep-

${ }^{3}$ Apparently, only the ventral pathway is mislead by this illusion; the dorsolateral pathway represents the size of the objects accurately (Jacob and Jeannerod 2003). So, a better description of the case would say that there is a mechanism in the ventral pathway that reliably misrepresents size. 
resentations. Nevertheless, there is a representation type $\mathrm{R}$ (the state that misrepresents the size of inner circle in the Ebbinghaus scenario) that reliably and systematically misrepresents a certain configuration. This state R, which reliably misrepresents an inexistent size of certain circles, is a by-product of the representational system that has earned its keep in evolution. This is a sort of case involving strong reliable misrepresentation that can be perfectly accommodated within teleosemantics.

There is a second way teleosemantics can account for cases of strong reliable misrepresentation that Mendelovici does consider (although she utterly rejects this option for reasons that will become clear below). A given mechanism could produce P-involving representations because the organism was confronted with instances of $\mathrm{P}$ in the evolutionary past and, nevertheless, at a certain time t, P might not be instantiated any more. Toads (Bufo Bufo), for instance, dart on any elongated object moving at a certain velocity in the direction of its axis (Ewert 2004). If a toad is grown up in a laboratory, where all the moving black things it sees are nutritious pellets, this toad will be consistently and reliably misrepresenting all its life. We could even make the case more extreme by supposing that flies go extinct, so that flyhood is never instantiated again. In this case, all toads will be reliably misrepresenting flies. Also in this case, teleosemantics is fully compatible with cases of strong reliable misrepresentation.

Since neither reliable misrepresentation nor strong reliable misrepresentation can do the trick, Mendelovici needs a different kind of example. Indeed, she suggests an additional condition that should also be taken into account: the strong reliable misrepresentation must also be stable. She suggests that a reliable representation is stable when it lasts for a significant period of time. Accordingly, the proposal is that the sort of examples that might threaten teleosemantics concern cases of strong and stable reliable misrepresentation. She argues that the example of toads (Bufo Bufo) does not exemplify a case of strong and stable reliable misrepresentation, because this sort of misrepresentation is unstable. Since in the laboratory nutritious pellets (and not flies) help toads to survive, teleosemantics entails that toads will come to represent soon the presence of nutritious pellets, rather than the presence of flies. 
Now, the notion of stability appealed to here should be qualified in an important way: it is not obvious that teleosemantics cannot accommodate cases of strong and stable reliable misrepresentation, as far as this significant period of time is insufficient for evolution to take place. For instance, teleosemantics is compatible with an individual misrepresenting all its life (e.g. the toad in the laboratory discussed earlier). Unless a process of selection occurs, teleosemantics can accommodate cases of strong reliable misrepresentation lasting for a significant period of time. And we know that within a given population, many years or even centuries might pass by before evolution takes place. So, merely adding stability to strong reliable misrepresentation falls short of specifying a counterexample for teleosemantics: the relevant case Mendelovici is after should include a period of time long enough for evolution to take pace.

Now, prima facie it is hard to think of any example that can satisfy these conditions of stability, reliability and systematic misrepresentation. Mendelovici (10) helps us by describing the particular counterexample she has in mind. She claims that teleosemantics is incompatible with the following situation: a representation type $\mathrm{R}$ represents a property P, P correlates with Q and Q (but not P) explains why during a significant period of time the organism has acted successfully and why it was selected for. According to her, that would be a case in which $\mathrm{R}$ would be reliably misrepresenting $\mathrm{Q}$ as a P. Mendelovici argues this possible situation is ruled out a priori by teleosemantics. On teleosemantics, if instantiations of property $\mathrm{Q}$ explain the selection of the mechanism that produces $\mathrm{R}$, then $\mathrm{R}$ will be representing $\mathrm{Q}$ rather than $\mathrm{P}$. So teleosemantics entails that it cannot happen that $\mathrm{R}$ represents $\mathrm{P}$ and $\mathrm{Q}$ explains why R-representations have been selected for.

Mendelovici's objection, then, is that teleosemantics fails to account for cases of strong and stable reliable misrepresentation involving a process of evolution and in which the property that accounts for the selection of the mechanism (Q) is different from the represented property (P). At this point, I completely agree with Mendelovici. This, I think, is the only case teleosemantics cannot account for. The interesting question, however, is whether this example raises any difficulty for teleosemantics. Is this is an objectionable consequence of the theory? 
The answer, I think, is clearly negative. First, remember I showed that Mendelovici can only appeal to cases of strong and stable reliable misrepresentation involving a process of selection. Consequently, her objection is that teleosemantics is incompatible with the following case: $\mathrm{Q}$ is the property that causally explains the evolutionary success of having a representation $\mathrm{R}$, but $\mathrm{R}$ does not represent $\mathrm{Q}$ but P.

I agree this possibility is certainly ruled out by teleosemantics; according to the theory, R represents Q iff Q causally explains the selection of the mechanism producing R. However, that does not seem to be an unwelcome result, but just a different way of stating the theory. If $\mathrm{R}$ represents whatever feature explains its selection, it cannot happen that a feature explains its selection and it is not represented by R. Why should that be a problem?

The same point can be made in a different way. Every theory, teleosemantics a fortiori, is such that whatever meets the sufficient conditions for being an $\mathrm{F}$ according to a theory is an $\mathrm{F}$ according to the theory. This is just what sufficient conditions are. In teleosemantics, those sufficient conditions involve a process of reliability and stability for a period sufficient for selection of the sender-receiver configuration. Consequently, it is certainly true that teleosemantics rules out a case in which $\mathrm{Q}$ is the property that accounts for the selection of $\mathrm{R}$ and $\mathrm{R}$ does not represent $\mathrm{Q}$, but any theory giving sufficient conditions for being an $\mathrm{F}$ is incompatible with the presence of sufficient conditions without there being an F. Therefore, the fact that there is a case of reliable misrepresentation that is ruled out by teleosemantics should not be regarded as a problematic consequence of the theory. It is just a different way of formulating the key tenets of this approach. ${ }^{4}$

\footnotetext{
${ }^{4}$ This response is also available to any other version of teleosemantics. For instance, according to Neander's (1995) approach, content is utterly determined by the properties that the organism is able to discriminate. That is, on her view, toads represent something like there is a black moving thing. Now, certainly her theory excludes cases in which an organism represents $M$ and it cannot discriminate $\mathrm{M}$. That is an obvious consequence of the theory, but it is not clear why she should be worried about it.
} 


\section{Metaphysical commitments of teleosemantics}

I just argued that cases of reliable misrepresentation do not threaten teleosemantics. Nonetheless, I think there is a deep and intriguing issue underlying Mendelovici's reasoning that should be addressed by teleosemantics. The interesting question she is trying to raise (which, as I will argue below, she wrongly expressed in terms of reliable misrepresentation) is whether teleosemantics is compatible with certain anti-realist positions, e.g. color eliminativism. This is one of the problems pointed out by Mendelovici's paper I would like to turn to in the remainder. ${ }^{5}$

First of all, notice that, strictly speaking, teleosemantics as such is compatible with the denial of realism about any entity. For instance, teleosemantics can be true at a possible world $w$, even if no organism exists at $w$, or even if evolution has never taken place at $w$. What the theory claims is that there are representations at a world $w$ if and only if certain processes occur (involving some systems, natural selection and so on). So teleosemantics as such has no realist implications.

Mendelovici, however, tries to put some pressure on teleosemantic theories in that direction. She argues that if we accept teleosemantics and certain empirical claims, the theory has inadequate consequences concerning the debate between realism and anti-realism. In particular, she claims that accepting teleosemantics 'would force us to be realists about properties represented in nonsemantically successful conditions, where realism about property $\mathrm{P}$ is the view that P is instantiated' (Mendelovici: 18). Now, does teleosemantics force us to be realist about properties represented in nonsemantically successful conditions? I think the answer is affirmative, but again I doubt granting this point results in any problematic consequence for

\footnotetext{
${ }^{5}$ Mendelovici (16-7) puts forward another argument, which I think can be easily defeated given the results of the previous discussion. First she distinguishes veridicality from reliability: a mechanism is veridical if it yields the right result and it is reliable if it tends to produce the same result, regardless of whether it is veridical. Her 'psychological argument' claims that if a theory is unable to account for reliable misrepresentation, it cannot maintain the useful distinction between veridicality and reliability. Now, since I have already shown that teleosemantics can accommodate many cases of reliable misrepresentation, it should be obvious that it can also make this distinction.
} 
the theory. Let me explain.

In teleosemantics ' $\mathrm{P}$ is represented in non-semantically successful conditions' should be spell out as the claim that $\mathrm{P}$ was the property that accounted for the selection of the sender-receiver system. Hence, the problem should be cashed out as follows: if teleosemantics is right and $\mathrm{P}$ is the property that accounts for the existence and selection for the system, then one is committed to the (past) existence of P. ${ }^{6}$ Again, this conditional seems true, but also entirely plausible. If a property $\mathrm{P}$ accounted for the existence of the representational system, P must have been instantiated somewhere.

Indeed, this inference is not only plausible, but it describes a standard way of reasoning in science. A clear example can be found in research on arachnophobia (the fear of spiders and other arachnids). Some studies suggest that evolution might have equipped mammals with a strong predisposition to react fearfully to spiders (Öhman and Mineka 2001). One of the standard criticisms to this proposal is that only 0.1 percent of the 35.000 different kinds of spiders in the world are poisonous, so probably having a predisposition to react fearfully to spiders did not constitute any significant selective advantage for mammals (Gerdes et al. 2009). The debate, then, assumes that if humans possess a mechanism for producing fearful reactions to spiders, then a sufficient number of poisonous spiders must have existed in the past. Hence, scientists accept that if we are endowed with a mechanism for representing or behaving towards P, then P must have existed in the past. That looks like an impeccable scientific reasoning.

Similar arguments are also common in philosophy, e.g. in relation to radical conceptual nativism (Fodor 1975, 1998). Some people have suggested that human concepts like CARBURETOR or TELEVISION cannot be innate because if they were, we would have to accept that there were carburetors and televisions at the time our ancestors evolved (Sterelny 1989; Prinz 2002: 229). Again, it is assumed here that the truth of some claims about conceptual content

\footnotetext{
${ }^{6}$ It is worth pointing out that if teleosemantics is committed to realism about an entity, it is realism about an entity in the evolutionary past. As we saw, teleosemantics is compatible with the possibility of P-involving representations in cases where $\mathrm{P}$ has gone extinct.
} 
commits one to certain ontological claims.

Therefore, one should not try to devise a general argument against drawing metaphysical conclusions from theories of meaning and certain empirical claims about the current representational capacities of organisms. Many discussions in science and philosophy take for granted that if we have good reasons for thinking that an organism has build-in a mechanism for representing a set of properties $\mathrm{P}$, this set of properties $P$ existed in the past.

In order to make her objection to teleosemantics more compelling, Mendelovici focuses on a particular case, in which this general way of arguing seems to go astray: color. More precisely, she argues that if we accept teleosemantics, the following inference could be carried out:

P1 I have experiences of redness.

P2 My experiences of redness at least sometimes occur in (nonsemantically) successful conditions.

P3 If I have experiences of redness in (nonsemantically) successful conditions, then realism about redness is true.

C Realism about redness is true.

She claims that realism about a certain entity like colors should not be so easy to get, so given the strong intuitive support for P1 and P2, P3 (which directly derives from teleosemantics) should be abandoned. The objection, then, is that teleosemantics warrants certain inferences and conclusions in the realist debate that a theory of content should not allow.

Before directly addressing this particular version of the objection, let me clarify the first premise, which I think is unduly imprecise. P1 could be interpreted (at least) in three different ways: as a claim about representational content (I have experiences about redness), about phenomenal properties (I have experiences instantiating phenomenal redness) or about the two (I have an experience about redness instantiating phenomenal redness). Now, remember that this argument is supposed to make explicit a consequence of 
endorsing teleosemantics and this naturalistic approach is exclusively concerned with representational content (see Millikan 1984, Neander 2012). Teleosemantics as such is a theory of content, and it is silent concerning the relationship between representational content and phenomenal properties. Therefore, in that particular case, P1 should be read as stating that experiences are about redness. That is, $\mathrm{P} 1$ is a claim about representational content.

Indeed, notice if P1 were interpreted as stating that experiences instantiate a certain phenomenal property (redness), P1-P3 would not entail $\mathrm{C}$ without additional and controversial assumptions about the connection between phenomenal and representational properties, which are clearly not made by teleosemanticists and would require independent support.

Having clarified premise P1, let me argue why I think Mendelovici is right in her formulation of the inference, but (again) teleosemanticists can happily accept this result.

First of all, we saw that drawing certain ontological claims from a theory of meaning plus certain empirical claims is generally regarded as valid. So what is wrong with this reasoning? Some people will probably find this particular inference objectionable because of the a priori status of P1 and P2. Since P1 and P2 seem to be priori and $\mathrm{C}$ is clearly a posteriori, if we accept that P1-P3 entail C, we will be entitled to conclude a substantive and a posteriori claim about the world (color realism) from certain a priori claims and teleosemantics. I think this is precisely what worries Mendelovici, since she thinks that $\mathrm{P} 1$ can be established by introspection alone, $\mathrm{P} 2$ is uncontroversial and she accuses teleosemantics of enabling one to become a realist about a certain entity 'without any empirical examination of objects' (Mendelovici: 17). ${ }^{7}$

\footnotetext{
${ }^{7}$ Here is another quote where Mendelovici makes clear that her main objection concerns the fact that premises $\mathrm{P} 1$ and $\mathrm{P} 2$ are a priori while the conclusion is a posteriori: 'But if tracking theories are correct, then in order to establish realism about a represented property $\mathrm{P}$, we needn't check the world for evidence of instances of P. We can instead check ourselves for nonsemantically successful instances of the representation of P. (Mendelovici, forthcoming, 18; so also footnote 19). Nonetheless, let me point out that, at the same time, Mendelovici claims that she is not concerned about the a priori status of the premises (20). Again, if this is true, I fail to see why this inference is problematic.
} 
However, this is surely not warranted by teleosemantics. Teleosemantics is an externalist theory about content, so P1 and P2 are a posteriori claims through and through. What kind of property I am representing with a red experience and what kind of situations are nonsemantically successful conditions (i.e. what sort of situations accounted for the selection of the mechanism) are hard empirical questions that should be resolved by science. Consequently, even if teleosemantics is right, a considerable amount of empirical knowledge must be gathered before anything like $\mathrm{C}$ can be established. Certainly, if we accept that color-experiences are representations (as they probably are) then, teleosemantics is committed to there being a property they are supposed to represent. However, what kind of entity we are committed to is something that should be discovered by empirical research.

Of course, if one assumes that the content of mental states can be discovered through introspection alone (as Mendelovici seems to suggest in 18), then this claim is in tension with teleosemantics. In general, externalist theories threaten the privileged access we seem to have to the content of our own mental states. But we already knew that externalist theories (and teleosemantics among them) are in tension with certain internalist intuitions, so on this interpretation there is nothing new about Mendelovici's argument (see Boghossian 1997). Furthermore, notice that if this is what the argument intends to show, there is no specific objection to teleosemantics or tracking theories: any externalist theory of content has this difficulty. Accordingly, a defense will have to come from externalism, rather than from teleosemantics.

Finally, let me conclude by directly addressing Mendelovici's main question: if we assume teleosemantics and grant everything I accepted in this paper (including the inference from P1-P3 to C), is teleosemantics still compatible with color eliminativism? It clearly is. If science discovers that there is nothing our color experiences have been tracking, then teleosemantics has to say that the mechanism that produces our color experiences is not a representational mechanism. That is, it is possible that color experiences are not representational states. There are many alternative evolutionary explanations for the existence of the mechanism: evolutionary drift, sprandels, and so on. Of course, I am not saying that this is an attractive view; 
but the objection was based on the incompatibility of teleosemantics and color eliminativism, not on the plausibility of the latter. What we had to show is that accepting teleosemantics and granting the validity of the inference described earlier does not make teleosemantics incompatible with color eliminativism. ${ }^{8}$

Finally, let me make a general comment on Mendelovici's dialectical strategy. In this paper, I have addressed the question of reliable misrepresentation and the question of color realism separately. But Mendelovici's strategy is to use the first debate in order to conclude something about the second. In particular, she argues that (1) teleosemantics is incompatible with color-experiences reliably misrepresenting and then that (2) if color eliminativism is true, then color-experiences reliably misrepresent. If (1) and (2) were true, then teleosemantics would be incompatible with color eliminativism. However, in the first part of the paper I showed that (1) is false and I just presented an argument suggesting that (2) is false as well. According to teleosemantics, if there are no colors (if there is nothing our color experiences have been tracking), then the mechanism producing color experiences is not a representational system and, consequently, color experiences are not representations. And, of course, if experiences do not represent anything, they cannot misrepresent either. So, Mendelovici is wrong in assuming that the only way for teleosemantics to be compatible with color eliminativism is by accommodating reliable misrepresentation. Consequently, the question about reliable misrepresentation and the question about color eliminativism should be clearly distinguished. This is the reason I have addressed (1) and (2) separately.

\section{Conclusion}

In conclusion, I argued that teleosemantics can account for most cases of reliable misrepresentation and that those particular instances

\footnotetext{
${ }^{8}$ Of course, I am not denying that certain views are incompatible with Teleosemantics. For instance, probably one cannot coherently hold at the same time that (1) teleosemantics is true, that (2) color experiences are representational, and that (3) there is nothing color experiences have been tracking in the evolutionary past. However, prima facie this sort of incompatibilities do not seem to be a problem. Thanks to a referee for pressing me on this issue.
} 
that cannot be accommodated do not pose any significant problem for the theory. On the other hand, Mendelovici's general objection against drawing ontological conclusions from a theory of content (plus certain empirical claims) seems to be wrong-headed. In sum, teleosemantics is neither threatened by cases of reliable misrepresentation, nor by any metaphysical consequence of the theory. ${ }^{9}$

\author{
Marc Artiga \\ Departament de Filosofia \\ Facultat de Lletres \\ Universitat de Girona \\ Plaça Ferrater i Mora, 3 \\ 17071 Girona \\ marc.artiga@gmail.com
}

\title{
References
}

Boghossian, Paul. 1997. What an externalist can know a priori. Proceedings of the Aristotelian Society 97 (2): 161-175.

Comer, Christopher and Leung, Vicky. 2004. The Vigilance of the Hunted: Mechanosensory-Visual Integration in Insect Prey. In Complex Worlds from Simpler Nervous Systems, ed. by F. R. Prete. Cambridge: MIT Press.

Evans, Christopher and Evans, Linda. 1999. Chicken food calls are functionally referential. Animal Behavior 58(2):307-319.

Ewert, Jörg-Peter. 2004. Motion perception shapes the visual world of amphibians. In Complex Worlds from Simpler Nervous Systems, ed. by F. R. Prete. Cambridge: MIT Press.

Fodor, Jerry. 1975. The Language of Thought, Cambridge, Cambridge: Harvard University Press.

Fodor, Jerry. 1998. Concepts: Where Cognitive Science Went Wrong. Oxford: Oxford University Press.

Fodor, Jerry. 1990. A Theory of Content And Other Essays. Cambridge: MIT Press.

Gerdes, A., Uhla, G., Alpersa, G. 2009. Spiders are special: fear and disgust evoked by pictures of arthropods. Evolution and Human Behavior, 30: 66-73.

Godfrey-Smith, Peter. 1996. Complexity and the Function of Mind in Nature. Cambridge: Cambridge University Press.

Jacob, Pierre and Jeannerod, Marc. 2003. Ways of Seeing: The Scope and Limits of Visual Cognition. Oxford: Oxford University Press.

Mendelovici, Angela. Forthcoming. Reliable misrepresentation and tracking the-

9 I would like to thank Manolo Martínez, Miguel Àngel Sebastian, David Pineda and an anonymous referee for helpful comments and incisive criticisms. This work was supported by the scholarship BES-2008-005255 from the Spanish Ministry of Economy and Competitivity (MEC), the Research Projects 'The Naturalization of Subjectivity' (ref. FFI2010-15717), 'Modal Aspects of Materialist Realism' (ref. HUM2007-61108) and Consolider-Ingenio project CSD200900056. 
ories of mental representation, Philosophical Studies.

Millikan, Ruth. 1984. Language, Thought and Other Biological Categories. Cambridge: MIT Press

Millikan, Ruth. 1993. White Queen Psychology and Other Essays for Alice, Cambridge: MIT Press.

Neander, Karen. 1995. Malfunctioning and Misrepresenting. Philosophical Studies 79: 109-141.

Neander, Karen. 2012. Teleological Theories of Mental Content. Stanford Encyclopedia of Philosophy.

Öhman Arne and Mineka, Susan. 2001. Fears, phobias, and preparedness: Toward an evolved module of fear and fear learning. Psychological Review 108(3): 483-522.

Papineau, David. 1993. Philosophical Naturalism. Cambridge: Basil Blackwell.

Prinz, Jesse. 2002. Furnishing the Mind: Concepts and their perceptual basis. Cambridge: MIT Press.

Skyrms, Bryan. 2010. Signals, Evolution and Learning. Oxford: Oxford University Press.

Sterelny, Kim. 1989. Fodor's Nativism, Philosophical Studies 55 (2): 119-141. 